\title{
Density variations in dry granular avalanches
}

\author{
Louis Bugnion · Marius Schaefer • Perry Bartelt
}

Received: 1 June 2011 / Published online: 25 July 2013

(C) Springer-Verlag Berlin Heidelberg 2013

\begin{abstract}
Dry granular avalanches exhibit bulk density variations. Understanding the physical mechanisms behind these density variations is especially important in the study of geophysical flows such as snow and rock avalanches. We performed small-scale chute experiments with glass beads to investigate how bulk density changes, measuring velocity profiles, flow height and basal normal stress in an Eulerian measurement frame. The chute inclination and the starting volume of glass beads were systematically varied. From the flow height and basal normal stress data, we could compute the depth-averaged density at the measurement location during the passing of the avalanches. We observed that the depth-averaged density is not constant, varying with chute inclination and starting volume. Furthermore, the depthaveraged density varies from the head to the tail within a single avalanche. We model changes in density by accounting for the energy associated with the velocity fluctuations of the grains, the density and the velocity fluctuations being related by the constitutive relation for the normal stress. We propose expressions for the conduction and decay coefficients of the fluctuation energy which allow us to model the observed density variations in the experiments.
\end{abstract}

Keywords Density measurement .

Finite-sized granular avalanches . Glass beads .

Chute experiment

L. Bugnion · P. Bartelt ( $ه)$

WSL Institute for Snow and Avalanche Research SLF, Davos Dorf, Switzerland

e-mail: bartelt@slf.ch

M. Schaefer

Instituto de Ciencias Físicas y Matemáticas, Facultad de Ciencias,

Universidad Austral de Chile, Valdivia, Chile

\section{Introduction}

Snow and rock avalanches are two examples of hazardous, finite-sized granular flows occurring in the natural environment. Engineers often apply flow models to simulate velocities, impact pressures and runout distances in order to delineate land into safe/unsafe zones [1]. Depth-averaged SavageHutter type models are typically used for this purpose [2]. For simplicity, the density of the flowing material is assumed to be constant from initiation to deposition. However, density variations in both natural and experimental flows are known to exist. Density time series in small-scale snow avalanches were recorded using a capacitance sensor and qualitative statements about the change in density from one flow region to another could be made [3]. The measured mean density directly at the head is lower than the density in the body or tail of the avalanche [4]. Impact pressure measurements of large, real scale avalanches corroborate this result as they sometimes show a "dilute" head, followed by a much denser core [5]. Nonetheless, direct density measurements in natural flows remain difficult and are opposed by numerous technical difficulties. One fundamental problem is that the granules in natural flows range over several orders of magnitude up to the meter scale [6]. Thus, to determine the bulk density in real scale geophysical flows requires density probes that capture density variations in volumes on the order of the flow height that is several meters.

To learn about the physical processes that take place in natural granular flows, small-scale experiments are useful. Shear cell experiments-including the original experiments of Bagnold [7] - are performed under constant volume, therefore preventing a change in density, but allowing for an increase in normal stress [8]. The measured normal stress increase is often merged into the more general problem of assessing the granular rheology of the material. The 
concept of dilatancy, defined as the volume increase (or density decrease) of a granular material subjected to a shear deformation, is often put forward to explain variations of the shear resistance $[9,10]$. Dilatancy may also be related to normal stress dependent shear resistance, implemented in common flow models $[2,11,12]$. In granular flows down inclined chutes, the flow volume is not fixed since the position of the free surface can vary. However direct measurements of density in chute experiments are scarce and no consensus emerges from the available data. Depth-averaged density in steady and uniform flows are either found to be almost constant [9] or to vary significantly [13] for similar ranges of chute inclinations and flow height to grain diameter ratios $h / d$. Numerical simulations of steady and uniform free surface flows within periodic boundaries predict non-constant density [14]. The shape of the density profile is reported to depend on the ratio $h / d$ and on the slope inclination. Furthermore the depth-averaged density value varies with the slope inclination. For $h / d \approx 10$ the density varies over the whole flow depth whereas it varies only close to the bed and free surfaces for $h / d>40$ and it is constant in the intermediate flow region. Models accounting for non-constant density in dry granular flows generally include a fluctuation energy equation in the system of governing equations. Constitutive relations for the internal stresses and the coefficients of the fluctuation energy equation either are postulated based on heuristic arguments $[15,16]$ or are computed using kinetic theory $[17,18]$ or combine both approaches [19]. Kinetic theory calculations assume binary, short duration contacts between grains and thus only apply to collisional flows.

In this work we present velocity and depth-averaged density measurements of finite-sized, unsteady granular avalanches in small-scale chute experiments. Accurate measurements of the basal normal stress and of the flow height allow for the determination of the depth-averaged density from the head to the tail of the avalanche. A high-speed camera records the flow through one of the sidewalls and the evolution of the velocity profile during the passing of the flow is obtained using a pattern matching algorithm [20]. We performed series of experiments systematically varying the chute inclination $\left(22^{\circ}, 24.5^{\circ}, 27^{\circ}, 29.5^{\circ}\right)$ and the starting volume (151 and 301). In all experiments we used the same granular material (1.4 mm glass beads) and the same chute with a rough bed surface and smooth sidewalls. In the second part of the article, we develop a simple model based on mass, momentum and fluctuation energy conservation equations for shallow, two-dimensional flows. We propose constitutive relations for the internal stresses and for the coefficients of the fluctuation energy equation (conduction and decay) following a phenomenological approach similar to the ones in $[15,16]$. The system of governing equations is completed by boundary conditions for the fluctuation energy and for the normal stress at the bed and free surfaces. The momentum and fluctuation energy equations are integrated numerically at the location of the camera during the passing of the flows and the results are compared with the depth-averaged density measurements.

\section{Experiments}

\subsection{Setup}

We performed the experiments on a $2 \mathrm{~m}$ long, $0.2 \mathrm{~m}$ wide chute with variable inclination (Fig. 1). Perpendicular sidewalls made of plexiglas bound the flow laterally. The bed surface of the chute is covered with sand paper with $0.1 \mathrm{~mm}$ (P150) roughness. The friction of the bed surface was therefore much higher than the one of the sidewalls. We define a two-dimensional reference plane along the chute centerline with the $x$-axis parallel to the chute length and directed towards the lower end of the chute and a $y$-axis perpendicular to the $x$-axis in the plane of the sidewalls directed opposite to the bed surface (Fig. 2). The origin of the coordinate system is set at the trap door. The chute inclination is parameterized by the angle $\theta$. Volumes of 15 and 301 of glass beads were released at four different chute inclinations: $22^{\circ}, 24.5^{\circ}, 27^{\circ}$ and $29.5^{\circ}$. The glass beads have a mean diameter $d$ of $1.4 \mathrm{~mm}$ with $92.5 \%$ of the beads having a diameter ranging between 1.18 and $2 \mathrm{~mm}$. The range of inclination angles is centered around the angle of repose of the glass beads, which was measured to be $25^{\circ}$. At the location $x=1.5 \mathrm{~m}$, the basal normal stress $N(t)$ is measured over a rectangular surface of $50 \mathrm{~cm}^{2}$ built in the bed surface. The force plate consists of a single point load cell with a measurement frequency of $2 \mathrm{kHz}$. Calibration tests were performed using static loads. At the same downstream location $x=1.5 \mathrm{~m}$, at the sidewall, the velocity component in the $x$-direction $u(y, t)$ is measured from the recordings of the high-speed camera. A pattern matching algorithm [20] is used to compute the velocity profiles during the passing of the granular avalanche. We determine the evolution of the flow height $h(t)$, that is defined as the $y$-coordinate of the free surface at the location of the camera, from the same recordings using a grey level threshold value.

\subsection{Results}

In Figs. 3 and 4, the flow height and the basal normal stress are plotted versus time for the four different chute inclinations and the two starting volumes. The normal stress $p$ can be related to the flow height and to the density by the expression:

$$
p(y)=\int_{y}^{h} \rho\left(y^{\prime}\right) g \cos \theta \mathrm{d} y^{\prime}
$$



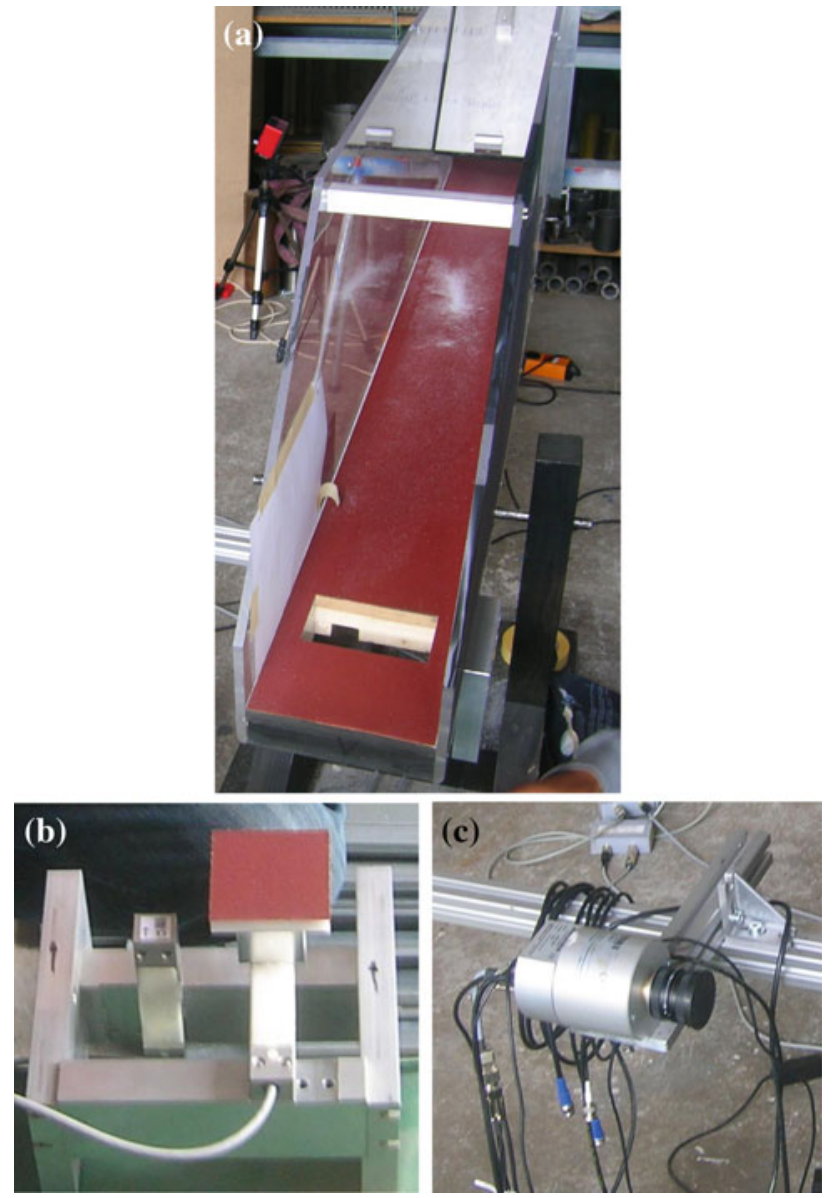

Fig. 1 Experimental setup. a Chute with plexiglas sidewalls, sand paper bed surface and location of the force plates. b Force plates. c High-speed camera

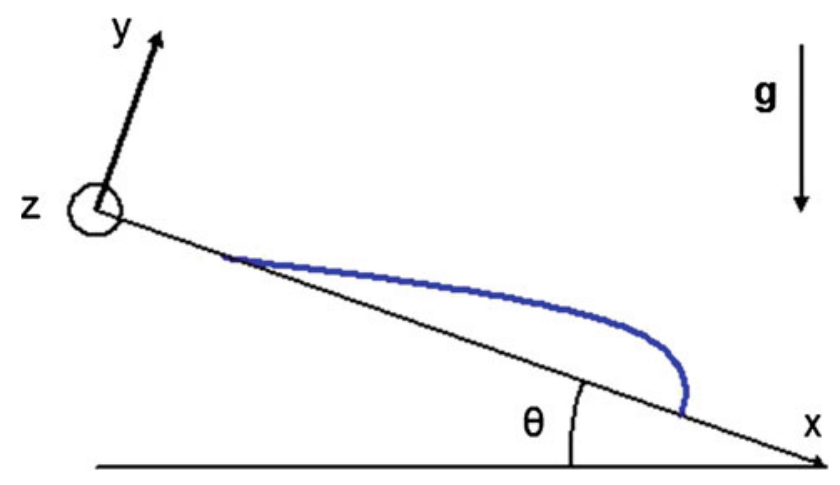

Fig. 2 Scheme of the chute with coordinate system

with $g$ the gravitational acceleration. Equation (1) represents the conservation of momentum in the $y$-direction in integral form for avalanches verifying the shallow flow assumption [2]. The basal normal stress $N$ is equal to the normal stress evaluated at the bed surface $y=0$ :

$p(y=0)=N=\bar{\rho} g \cos \theta h$
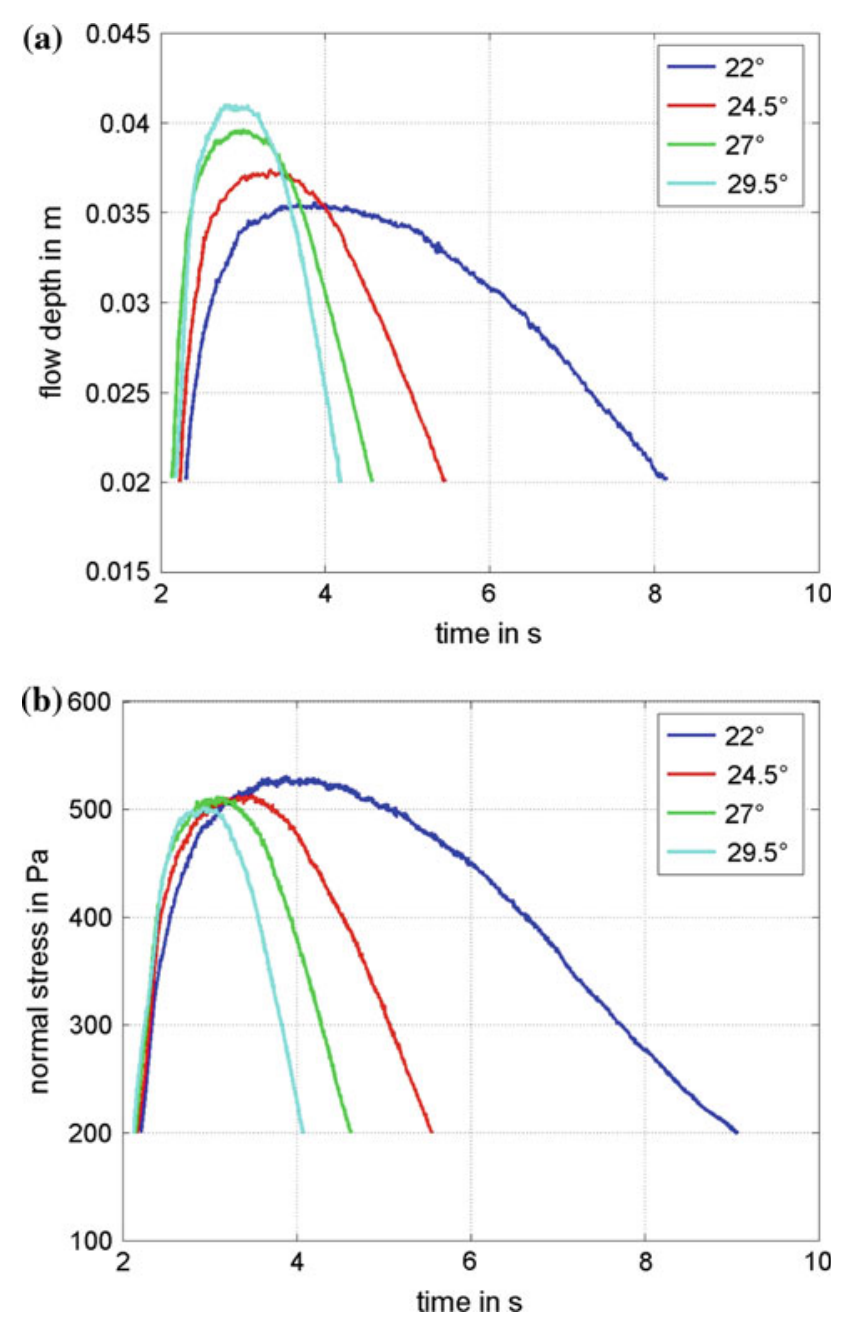

Fig. 3 Flow height (a) and basal normal stress (b) versus time for four different chute inclinations with 301 starting volume measured at the chute location $x=1.5 \mathrm{~m}$. The flow height data was truncated below $0.02 \mathrm{~m}$ for the 301 starting volume. Below this value, in the very head and in the very tail, the flow height could not always be determined precisely due to beads saltation at the free surface. The basal normal stress data was truncated below $200 \mathrm{~Pa}$ for the 301 starting volume in agreement with the truncation of the flow height data

with $\bar{\rho}$ the depth-averaged density:

$\bar{\rho}(t) \equiv \frac{1}{h} \int_{0}^{h} \rho \mathrm{d} y$.

In (2) we note that if the depth-averaged density is constant, the basal normal stress is proportional to the flow height (for a given chute inclination). In our experiments the depth-averaged density is found not to be constant during the passing of the flow. It is lower in the head and in the tail than in the body (Fig. 5a, b). If the chute inclination is increased, the depth-averaged density decreases in all flow regions. Likewise by reducing the starting volume from 30 to 151 , we observe that the depth-averaged density decreases 

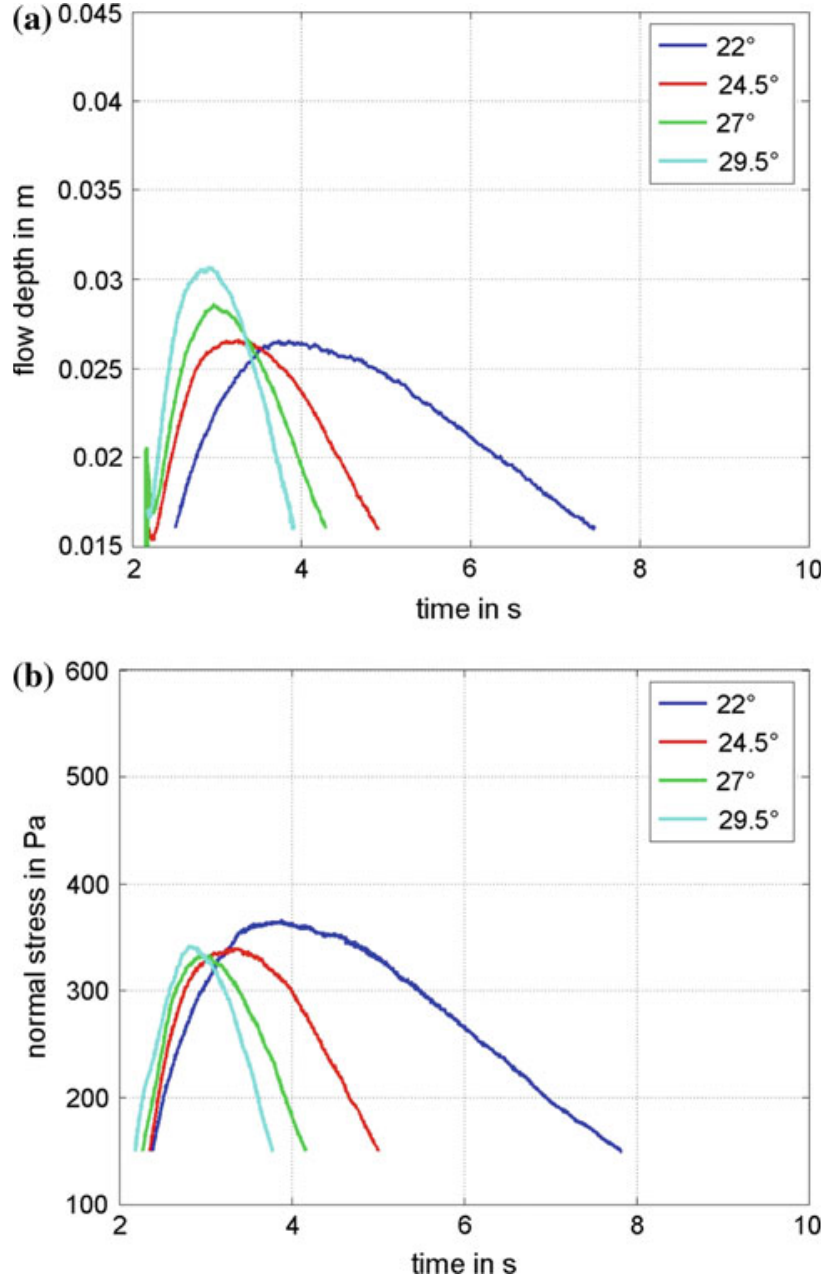

Fig. 4 Flow height (a) and basal normal stress (b) versus time for four different chute inclinations with 151 starting volume measured at the chute location $x=1.5 \mathrm{~m}$. The flow height data was truncated below $0.016 \mathrm{~m}$ for the 151 starting volume. The basal normal stress data was truncated below $150 \mathrm{~Pa}$ for the 151 starting volume

in all flow regions. For a chute inclination of $22^{\circ}$ and a 301 starting volume, the value of the depth-averaged density in the flow body is close to the "tapped density" value equal to $1,660 \mathrm{~kg} / \mathrm{m}^{3}$ (0.69 volume fraction). The "tapped density" was measured after pouring the material and vibrating the material container. After pouring the material the "poured density" was equal to $1,590 \mathrm{~kg} / \mathrm{m}^{3}$ ( 0.66 volume fraction). For a face centered cubic arrangement of monodisperse glass spheres $\left(\rho_{s}=2,400 \mathrm{~kg} / \mathrm{m}^{3}\right)$ with a diameter $d=1.4 \mathrm{~mm}$, the bulk density $\rho$ is given by the equation

$\rho=\rho_{s} \frac{\pi}{3 \sqrt{2}} \frac{d^{3}}{(l+d)^{3}}$.

With $l$, the distance between the surfaces of neighbouring grains, set to zero, Eq. (4) yields $\rho=1,780 \mathrm{~kg} / \mathrm{m}^{3}$ (0.74 volume fraction) whereas for a random close packing of monodisperse glass spheres, the density is equal
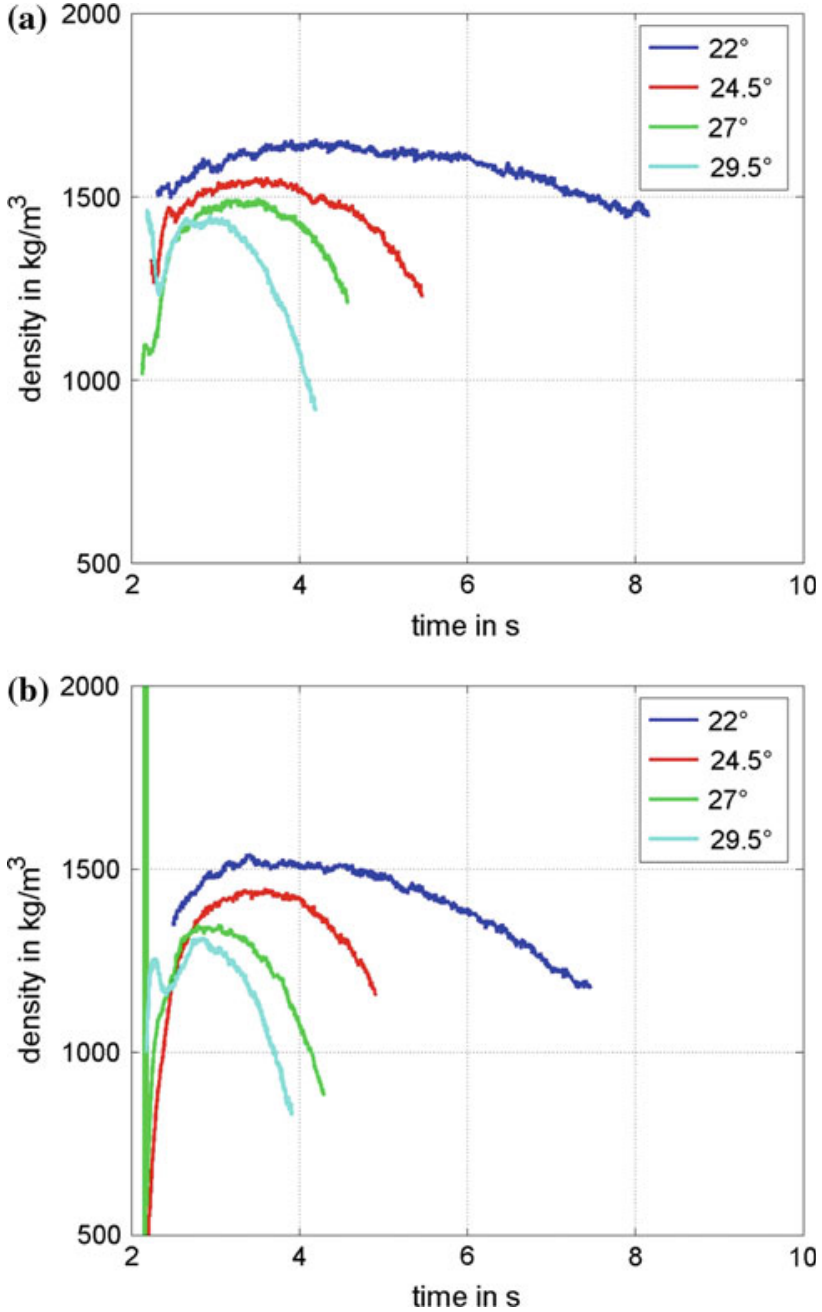

Fig. 5 Measured depth-averaged density versus time for four different chute inclinations and two starting volumes 301 (a) and 151 (b). We emphasize that the depth-averaged density is not measured simultaneously in different flow regions but successively. That is, we compute the depth-averaged density at the stationary point $x=1.5 \mathrm{~m}$, and do not move with the avalanche along the chute (Eulerian measurement frame)

to $1,540 \mathrm{~kg} / \mathrm{m}^{3}$ (0.64 volume fraction). The discrepancy between the "tapped" and "poured" density values and the density corresponding to a random close packing of glass spheres is explained by the slight polydispersity of the beads.

The mean shear rate, mean velocity and slip velocity are not constant during the passing of the flows (see Fig. 7a, b). The shear rate is approximately constant over the flow depth, i.e. the velocity profiles are close to linear (see Fig. 6), but it is not constant from head to tail. The mean shear rate is at a maximum in the head and at a minimum in the body. It increases again in the tail but not to the same level as in the head. The slip velocity shows a similar behaviour: it is at a maximum in the head and decreases to a minimum in the body. However it does not increase in the tail. 

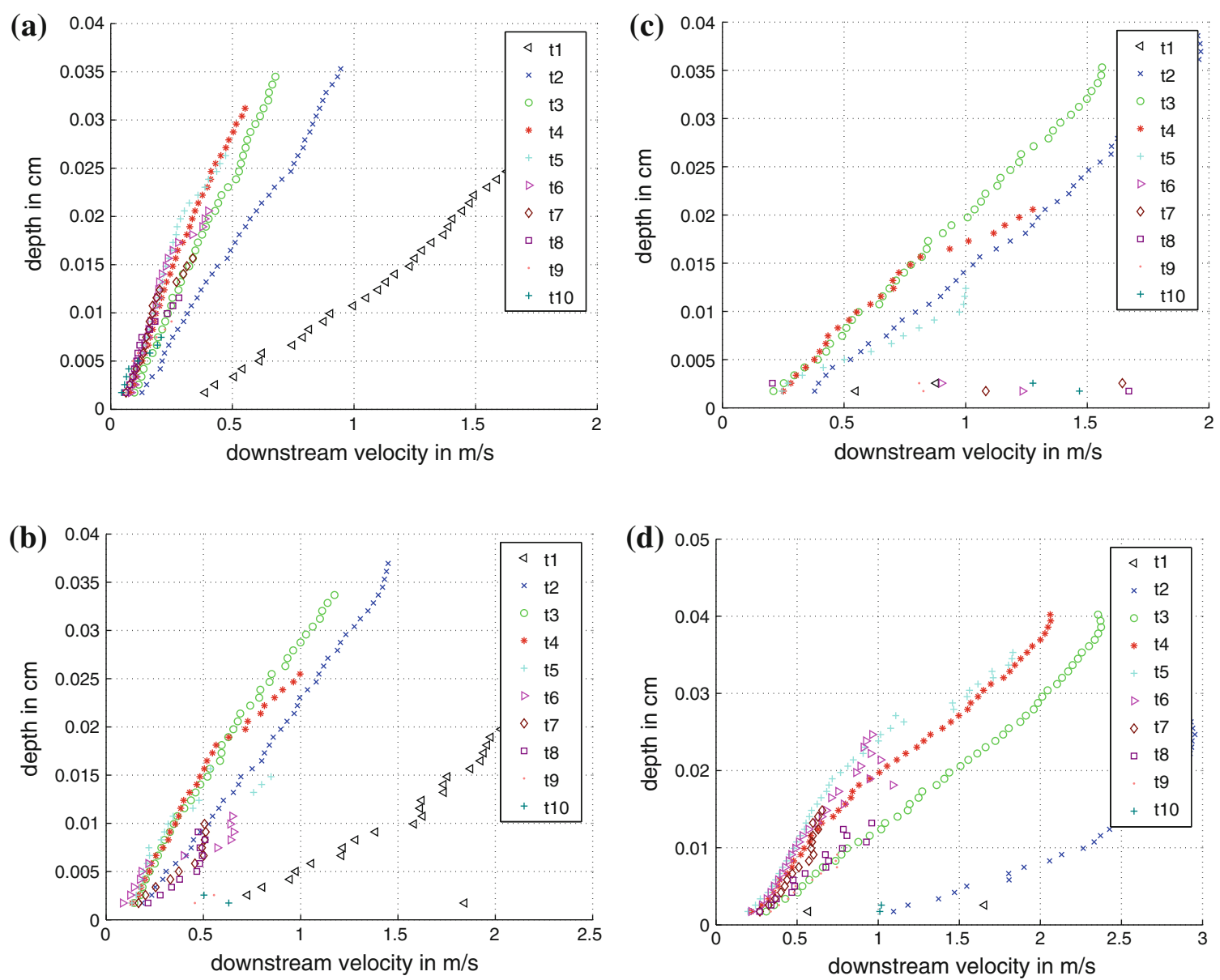

Fig. 6 Velocity profiles $u(y)$ at different instants of the flows at the location $x=1.5 \mathrm{~m}$ for the four chute inclinations $22^{\circ}(\mathbf{a}), 24.5^{\circ}(\mathbf{b}), 27^{\circ}(\mathbf{c})$, $29.5^{\circ}(\mathbf{d})$ and the 301 starting volume

\section{Model}

\subsection{Fluctuation energy equation}

In this section we develop a model with the aim of reproducing the evolution of the depth-averaged density measured experimentally. The velocity $\langle\boldsymbol{u}\rangle$ is defined as the mean of the velocities of the $N$ grains belonging to an average ensemble. An average ensemble must contain enough grains so that the averaged quantities are continuous and differentiable:

$\langle\boldsymbol{u}\rangle \equiv \frac{1}{N} \sum_{i=1}^{N} \boldsymbol{u}_{i}$.

The granular temperature $T$ is defined as the standard deviation of the grain velocities over an average ensemble divided by the dimension of the space [21]:

$T=\frac{1}{3}\left\langle(\langle\boldsymbol{u}\rangle-\boldsymbol{u})^{2}\right\rangle$.
We note that only translational velocities of the grains are considered. We simplify the notation by dropping the brackets of the velocity $\langle\boldsymbol{u}\rangle$. Anisotropic granular temperature was reported for glass beads in a specific experimental setup [22], however, for simplicity, we assume $T$ to be isotropic. The energy density $\rho T$ associated with the velocity fluctuations of the grains is termed the fluctuation energy. The molecular temperature of the granular material is irrelevant in the present problem since the energy associated with the molecular temperature of the grains is small compared to the kinetic and potential energies. We consider the granular avalanche as a continuum characterized by its density, velocity and velocity fluctuations and obeying the conservation equations for mass, momentum and fluctuation energy [23]. Besides we do not make a distinction between the grains and the interstitial air. The one-phase assumption is motivated by the small mean free path of the grains and by the low viscosity of air. The mass and momentum equations write:

$$
\frac{\partial \rho}{\partial t}+\nabla \cdot(\rho \boldsymbol{u})=0
$$


$\rho \frac{\partial \boldsymbol{u}}{\partial t}+\rho(\boldsymbol{u} \cdot \nabla) \boldsymbol{u}=\nabla \cdot \boldsymbol{\sigma}+\rho \boldsymbol{g}$

with $\sigma$ the stress tensor and $\boldsymbol{g}$ the gravitational acceleration vector. The fluctuation energy equation is specific to granular flows:

$$
\begin{aligned}
\rho \frac{\partial T}{\partial t}= & -\rho \boldsymbol{u} \cdot \nabla T-p \boldsymbol{\nabla} \cdot \boldsymbol{u}+\tau_{i k} \frac{\partial u_{i}}{\partial x_{k}} \\
& +\nabla \cdot(\kappa \rho \nabla T)-\rho \gamma
\end{aligned}
$$

with $\kappa$ the granular temperature conduction coefficient. We assume that the normal stress $p$ is sustained by the velocity fluctuations. As a consequence a volume change results in a variation of the fluctuation energy equal to $-p \boldsymbol{\nabla} \cdot \boldsymbol{u}$. Dissipative contacts between grains reduce the norm of the velocity vectors of the grains. The associated decay of fluctuation energy is accounted for by the coefficient $\gamma$. For experimental work on the decay of fluctuation energy see [24]. In Eq. (9) we assume that the stress tensor can be separated into a mean normal stress tensor and a stress deviator tensor:

$\boldsymbol{\sigma}=-p \boldsymbol{I}+\boldsymbol{\tau} \quad$ with $p=-\frac{1}{3} \operatorname{Tr}(\boldsymbol{\sigma})$.

The dissipative work rate done by the stress deviator tensor is the source of fluctuation energy. The contacts between grains resulting from the shear deformation change the direction of the velocity vectors of the grains and thus increase the fluctuation energy at the expense of the mean kinetic energy. To summarize, the first term on the right-hand side of Eq. (9) is the convective acceleration term, the second term accounts for the work done by the normal stress in case of volume change, the third term represents the production of granular temperature in case of shear deformation, the fourth term stands for the conduction of granular temperature similarly to thermal conduction (i.e. from regions with high $T$ to regions with low $T$ ), the last term accounts for the decay of granular temperature due to dissipative contacts between grains. We rewrite Eq. (9) in the case of a two-dimensional flow:

$$
\begin{gathered}
\rho \frac{\partial T}{\partial t}=-\rho\left(u \frac{\partial T}{\partial x}+v \frac{\partial T}{\partial y}\right)-p\left(\frac{\partial u}{\partial x}+\frac{\partial v}{\partial y}\right) \\
+\tau_{x y}\left(\frac{\partial u}{\partial y}+\frac{\partial v}{\partial x}\right) \\
+\kappa\left(\rho \frac{\partial^{2} T}{\partial x^{2}}+\frac{\partial \rho}{\partial x} \frac{\partial T}{\partial x}+\rho \frac{\partial^{2} T}{\partial y^{2}}+\frac{\partial \rho}{\partial y} \frac{\partial T}{\partial y}\right)-\rho \gamma .
\end{gathered}
$$

The stress deviator tensor is symmetric to ensure that it cancels for any uniform flow (in particular for a rotating uniform flow [25]) and the granular temperature conduction coefficient $\kappa$ is assumed to be a constant for simplicity. In $[15,17]$, the authors propose relations for the conduction coefficient that are functions of $\rho$ and $T$. We have implemented such relations without noticing significant changes in the results. Invoking scaling arguments valid for shallow flows, several terms in Eq. (11) can be neglected. Let us adimensionalize
Eq. (11). The new dimensionless variables are:

$$
\begin{aligned}
& \tilde{t}=t \sqrt{\frac{g}{L}}, \tilde{x}=\frac{x}{L}, \tilde{y}=\frac{y}{H}, \tilde{u}=\frac{u}{\sqrt{g L}}, \tilde{v}=v \sqrt{\frac{L}{g H^{2}}}, \\
& \tilde{\rho}=\frac{\rho}{\rho^{*}}, \tilde{T}=\frac{T}{T^{*}}, \tilde{p}=\frac{p}{\rho^{*} g H}, \tilde{\tau}_{x y}=\frac{\tau_{x y}}{\rho^{*} g H}, \tilde{\kappa}=\frac{\kappa}{\kappa^{*}}, \\
& \tilde{\gamma}=\frac{\gamma}{\gamma^{*}}
\end{aligned}
$$

with $g$ the norm of $g, L$ a typical flow length, $H$ a typical flow height, $\rho^{*}$ a typical flow density of the material, for example the "tapped" density, $T^{*}$ a typical flow granular temperature, $\kappa^{*}$ a typical conduction coefficient and $\gamma^{*}$ a typical decay coefficient. We rewrite Eq. (11) in terms of the new dimensionless variables introducing the shallowness parameter $\epsilon \equiv \frac{H}{L}$ :

$$
\begin{aligned}
\tilde{\rho} \frac{\partial \tilde{T}}{\partial \tilde{t}}= & -\tilde{\rho}\left(\tilde{u} \frac{\partial \tilde{T}}{\partial \tilde{x}}+\tilde{v} \frac{\partial \tilde{T}}{\partial \tilde{y}}\right)-\frac{g H}{T^{*}} \tilde{p}\left(\frac{\partial \tilde{u}}{\partial \tilde{x}}+\frac{\partial \tilde{v}}{\partial \tilde{y}}\right) \\
& +\frac{g H}{T^{*}} \tilde{\tau}_{x y}\left(\frac{1}{\epsilon} \frac{\partial \tilde{u}}{\partial \tilde{y}}+\epsilon \frac{\partial \tilde{v}}{\partial \tilde{x}}\right) \\
& +\frac{\kappa^{*}}{L \sqrt{g L}} \tilde{\kappa}\left(\tilde{\rho} \frac{\partial^{2} \tilde{T}}{\partial \tilde{x}^{2}}+\frac{\partial \tilde{\rho}}{\partial \tilde{x}} \frac{\partial \tilde{T}}{\partial \tilde{x}}+\frac{1}{\epsilon^{2}} \tilde{\rho} \frac{\partial^{2} \tilde{T}}{\partial \tilde{y}^{2}}\right. \\
& \left.+\frac{1}{\epsilon^{2}} \frac{\partial \tilde{\rho}}{\partial \tilde{y}} \frac{\partial \tilde{T}}{\partial \tilde{y}}\right)-\frac{\gamma^{*} L}{T^{*} \sqrt{g L}} \tilde{\rho} \tilde{\gamma} .
\end{aligned}
$$

The flows investigated in this work match the shallow flow criteria i.e. the typical flow height $H$ is by far smaller than the typical flow length $L$. We have $H \approx 0.03 \mathrm{~m}$ and $L \approx$ $6 \mathrm{~m}$ yielding a shallowness parameter $\epsilon \approx 0.02$. Not knowing a priori all typical flow parameters $\left(T^{*}, \kappa^{*}, \gamma^{*}\right)$, it is only possible to compare some of the terms in the dimensionless fluctuation energy equation. The source term with the velocity gradient in the $x$-direction in Eq. (13) is smaller than the one with the gradient in the $y$-direction (factor $\epsilon^{-2}$ ) and thus can be neglected. On the third and fourth lines, the terms with spatial gradients of $\rho$ and $T$ in the $x$-direction are smaller than the terms with spatial gradients in the $y$-direction (factor $\epsilon^{-2}$ ) and can be dropped from further consideration. Since we assume that the normal stress is sustained by the velocity fluctuations of the grains, all terms on the first line of Eq. (13) have the same order of magnitude. Therefore, the remaining term on the second line of Eq. (13) is a factor $\epsilon^{-1}$ larger than the terms on the first line. Comparison with the remaining terms on the third and fourth lines is not possible a priori, but will be performed after the computation. The terms on the first line are thus neglected and the correctness of the simplification is assessed a posteriori. Returning to the dimensional formulation, Eq. (11) reduces to:

$\tau_{x y} \frac{\partial u}{\partial y}+\kappa\left(\rho \frac{\partial^{2} T}{\partial y^{2}}+\frac{\partial \rho}{\partial y} \frac{\partial T}{\partial y}\right)-\rho \gamma \approx 0$. 


\subsection{Constitutive relations}

The system of Eqs. (7), (8) and (9) for two-dimensional, shallow flows is under determined meaning that they are more unknown variables than equations. Thus, constitutive relations are needed to close the system. We look for constitutive relations specifying the shear stress $\tau_{x y}$, the pressure $p$ and the decay coefficient $\gamma$. For the shear stress, we use an approach similar to the one in [16]. We assume that it is controlled by contacts between grains resulting from the shear deformation. The term $\tau_{x y} \frac{\partial u}{\partial y}$ represents the energy per unit of time and volume dissipated by frictional and inelastic contacts between grains in a flow region sheared at a rate of $\frac{\partial u}{\partial y}$. We argue that it scales with the contact rate per grain times the number of grain per unit volume times the energy lost per contact. We moreover assume that the energy lost per contact is proportional to the relative velocity of the contacting grains (equal to $d \frac{\partial u}{\partial y}$ in average) squared times the grain mass. We obtain:

$\tau_{x y} \frac{\partial u}{\partial y} \propto \frac{\rho}{l}\left(d \frac{\partial u}{\partial y}\right)^{3}$.

The above expression holds in the collisional regime in which binary and short-duration contacts are dominant. In the following we make the assumption that it is also valid in the range of shear rate, density and normal stress investigated in this work. Equation (15) resembles the constitutive relation proposed in [9] for steady, dense granular flows. In [15], the shear stress scales with the contact rate $\frac{\sqrt{T}}{l}$ valid for a uniform flow with non-zero $T$ times an energy loss per contact proportional to $\left(\frac{\partial u}{\partial y}\right)^{2}$ that holds for a simple shear flow with zero $T$. The argument is inconsistent from a micromechanical point of view even though it introduces the same coupling between granular temperature and shear rate as in [17]. We use a similar approach for the decay coefficient $\gamma$. For a face centered cubic arrangement of spherical grains, the mean distance between neighbouring grains $l$ is given by Eq. (4). The energy per unit time and volume dissipated by grain contacts in a uniform flow region (the relative velocity of grains is proportional to $\sqrt{T}$ ) scales with the contact rate per grain times the number of grains per unit volume times the energy lost per contact. It yields:

$\rho \gamma \propto \frac{\rho}{l} T^{\frac{3}{2}}$

It is straightforward that if $\sqrt{T}<d \frac{\partial u}{\partial y}$, the production of fluctuation energy will dominate the decay. In the opposite case $\sqrt{T}>d \frac{\partial u}{\partial y}$ the decay will generally exceed the production. The decay and the production (through the shear stress) of fluctuation energy are largely influenced by the friction and the inelasticity of the grains. In the ideal case of frictionless and elastic grains $\gamma=0$. A parameter describing the degree of friction and inelasticity of the grains could be introduced in Eqs. (16) and (15). However since the granular material is the same in all experiments, we assume that the grain properties are included in the dimensionless proportionality coefficients $\tau_{0}$ and $\gamma_{0}$ making the proportionality signs in Eqs. (15) and (16) explicit. The normal stress $p$ is assumed to be sustained by the velocity fluctuations of the grains. They are responsible for the normal component of the relative velocity of the grains during contacts. The normal stress is defined as the momentum exchanged per unit of time and surface. We quantify it as the contact rate per grain times the number of grains per unit surface times the momentum exchanged per contact. We obtain:

$p \propto \frac{l+d}{l} \rho T$.

We remind that Eq. (1) states that the normal stress is in equilibrium with the weight of the overburden material. Equation (1) does not always apply though, even for static cases. In certain configurations (piles of grains with a typical height $H$ larger than the typical width $W$ for example) granular materials do not exhibit depth dependent normal stress due to boundary effects (arching) [21]. In the present case we argue that Eq. (1) holds since $H$ is small compared to $W$ and $L$ and since the friction of the sidewalls is small.

\subsection{Computation}

We compute the depth-averaged density $\bar{\rho}(t)$ during the passing of the flow at the location $x=1.5 \mathrm{~m}$. To that purpose we solve the system of coupled partial differential equations made up by the momentum equation in the $y$-direction (differential form of Eq. (1)) and the fluctuation energy equation in Eq. (14) in which we introduce the constitutive relations (15) and (16):

$\frac{\partial p}{\partial y}=-\rho g \cos \theta$.

$\tau_{0} \frac{\rho}{l}\left(d \frac{\partial u}{\partial y}\right)^{3}+\kappa\left(\rho \frac{\partial^{2} T}{\partial y^{2}}+\frac{\partial \rho}{\partial y} \frac{\partial T}{\partial y}\right)-\gamma_{0} \frac{\rho}{l} T^{\frac{3}{2}} \approx 0$.

The density is the solution of the cubic equation obtained by eliminating $l$ in Eqs. (4) and (17) with $p_{0}$ the dimensionless proportionality coefficient making Eq. (17) explicit:

$\rho\left(\frac{p}{p-p_{0} \rho T}\right)^{3}=\rho_{s} \frac{\pi}{3 \sqrt{2}}$.

In order to solve the second order partial differential equation in Eq. (19) boundary conditions are needed for the granular temperature and the granular temperature gradient in the $y$ direction. We assume that the granular temperature at the bed surface is proportional to the square root of the slip velocity

$T(y=0, t)=\xi \sqrt{u_{s}}$ 
where $\xi$ is a constant that is a function of the bed surface and glass beads properties. We justify the form of the boundary condition by noting that the maximum values of the normal stress at the location of the force plate are approximately the same for a given starting volume independently of the chute inclination (see Figs. 3, 4). Thus, if there is a dependency of the basal shear stress on the basal normal stress, it is the same for all flows (for a given starting volume). Considering a flow at the location of the force plate at the time of maximum normal stress as a sliding block with constant velocity $u_{s}$, the basal shear stress must be equal to the component of the flow weight in the $x$-direction. From the slip velocity measurements, we find that the component of the weight in the $x$-direction is proportional to the square root of the slip velocity $\tau_{x y}(y=0)=\bar{\rho} g \sin \theta \propto \sqrt{u_{s}}$. The boundary condition is derived by assuming that the velocity fluctuations at the bed surface scale with the work of the basal shear stress. At the free surface we assume that the granular temperature gradient in the $y$-direction is equal to zero $\frac{\partial T}{\partial y}(y=h, t)=0$. An additional boundary condition is needed to solve the first order partial differential equation in Eq. (18). We choose the traction free condition at the free surface $p(y=h)=0$. For the computation we approximate the shear rate by the mean shear rate over the flow depth $\frac{\partial u}{\partial y}(y, t)=\frac{\partial u}{\partial y}(t)$. We consider the measured mean shear rate values $\frac{\partial u}{\partial y}$ and slip velocity values $u_{s}$ plotted in Fig. 7a, b. The coupled partial differential equations in Eqs. (18) and (19) are solved numerically in an iterative way using the forward Euler method. For details of the discretization of the system of equations, we refer to the "Appendix". In the flow bulk $T$ is initialized to one half of the $T$ value at the bed surface and $\rho$ is initialized to $1400 \mathrm{~kg} / \mathrm{m}^{3}$. The iteration process is continued until the depth-averaged density converges (around 10 iterations). The input parameters are: the basal normal stress (the initial flow height is determined from the basal normal stress and the initial density value of $1400 \mathrm{~kg} / \mathrm{m}^{3}$ ), the mean shear rate, the slip velocity, the ratios of coefficients $\frac{\tau_{0}}{\kappa}=0.625 \mathrm{~s} / \mathrm{m}^{2}$ and $\frac{\gamma_{0}}{\kappa}=1.25 \mathrm{~s} / \mathrm{m}^{2}$, the coefficient $p_{0}=1$ and the coefficient $\xi=0.015(\mathrm{~m} / \mathrm{s})^{\frac{3}{2}}$. The values of the coefficients $\frac{\tau_{0}}{\kappa}, \frac{\gamma_{0}}{\kappa}$ and $\xi$ were chosen following a best-fit approach for the flow with $22^{\circ}$ chute inclination and 301 starting volume. They were then kept constant for the computations of the other flows.

In Fig. 8 the computed depth-averaged density during the passing of the flows ( 30 and 151 starting volumes and four different chute inclinations) are plotted (symbols). They are in good agreement with the evolution of the measured depthaveraged density (solid lines). In the flow head the model underestimates the depth-averaged density in particular for the flows with $22^{\circ}$ chute inclination. We remark that the passing of the flow head is very short (less than a second) during which the flow height and the basal normal stress vary from zero to their maximum values. Small systematic errors in
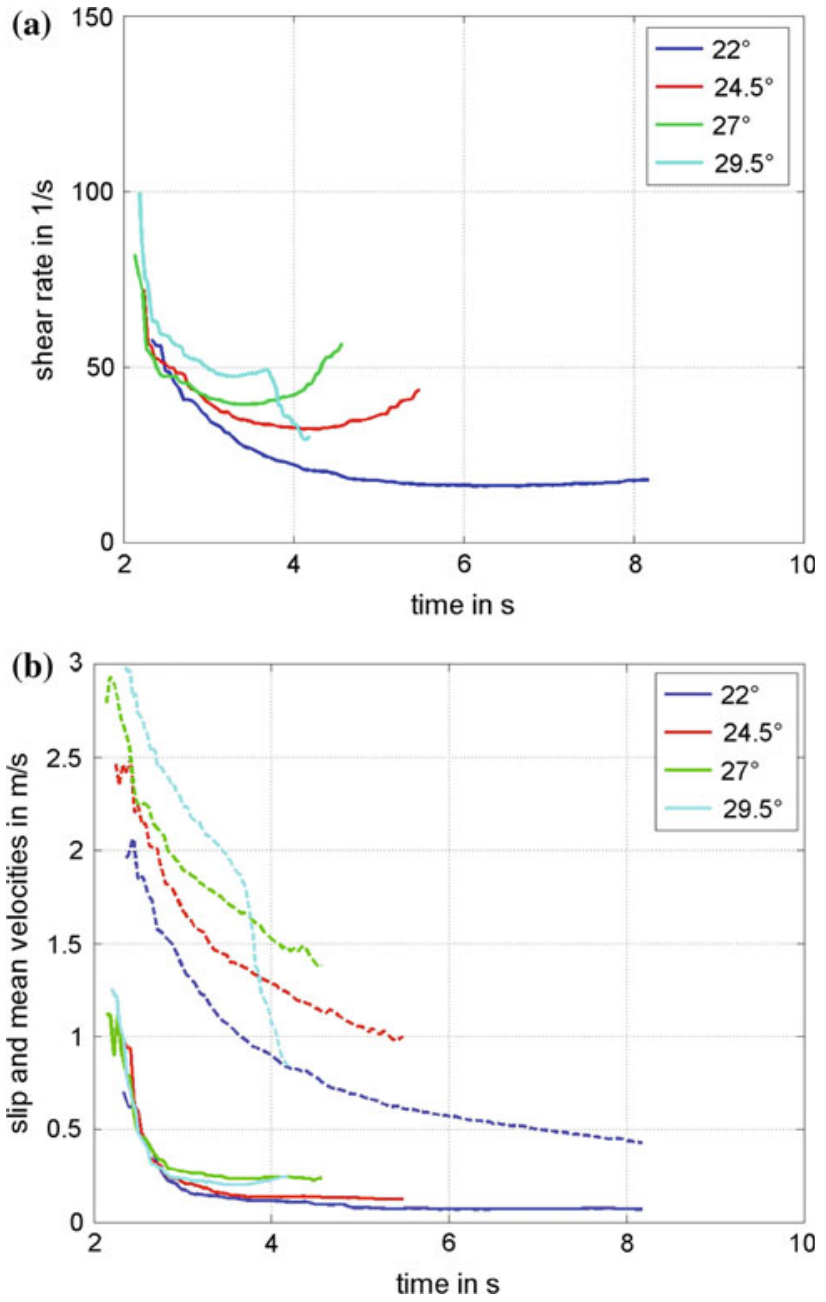

Fig. 7 Mean shear rate (a), mean velocity (dashed lines) and slip velocity (solid lines) (b) versus time at the location $x=1.5 \mathrm{~m}$ for the four chute inclinations and the 301 starting volume

the flow height or basal normal stress data would result in large uncertainties in the measured depth-averaged density values (ratio of small quantities). The agreement of the computed depth-averaged density with the measured one in the flow head is thus difficult to assess. The model predicts low depth-averaged density in the flow head due to high shear rate and slip velocity. In the flow body and tail the agreement between measurement and computation is good.

\section{Discussion}

The evolution of the measured depth-averaged density is reproduced using a model that accounts for the fluctuation energy of the grains. The normal stress is assumed to be sustained by the velocity fluctuations of the grains and the shear stress to be proportional to the density and to the shear rate squared. The granular temperature decay coefficient is 

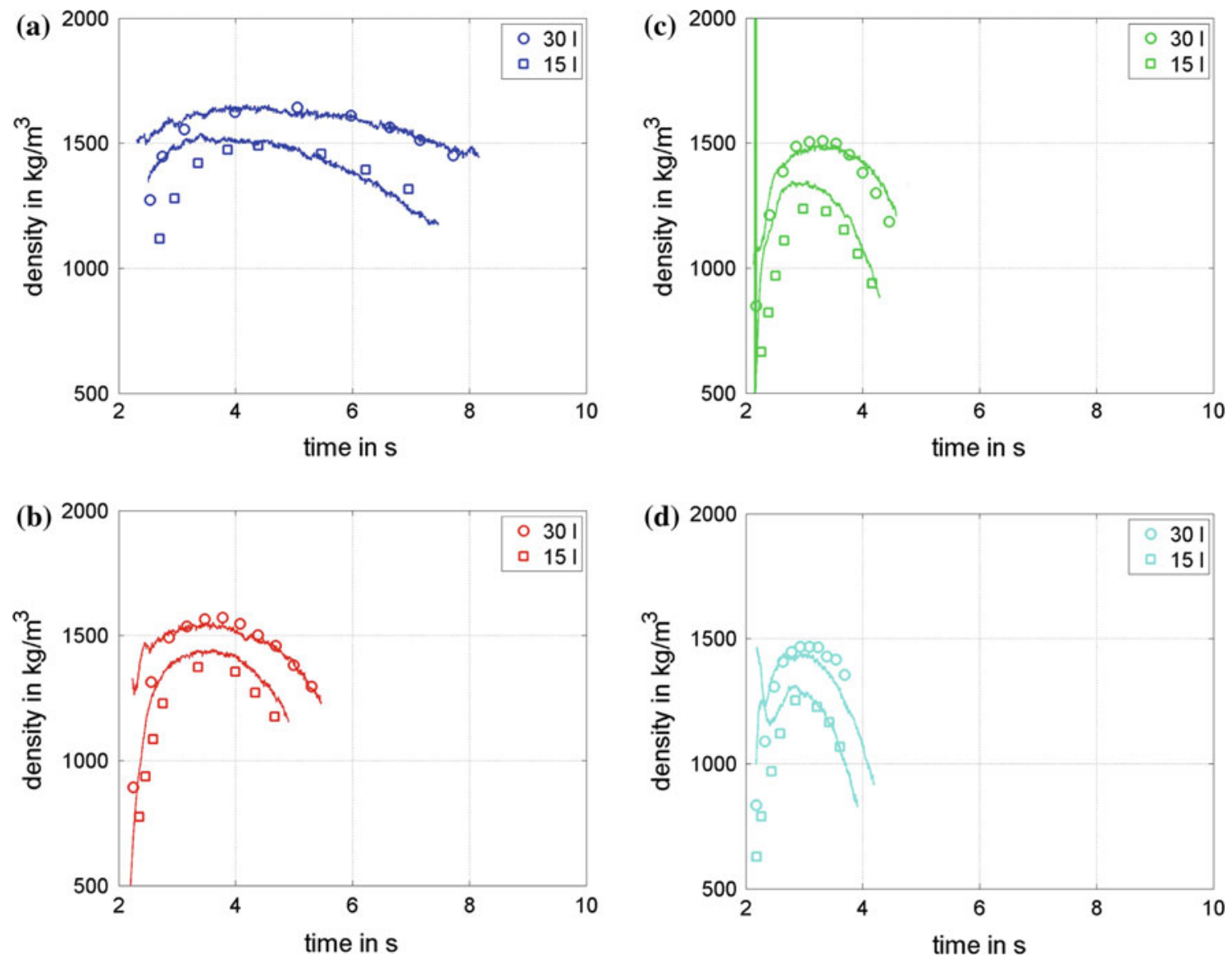

Fig. 8 Computed (symbols) and measured (solid lines) depth-averaged density versus time for flows with four different chute inclinations $22^{\circ}$ (a), $24.5^{\circ}(\mathbf{b}), 27^{\circ}(\mathbf{c}), 29.5^{\circ}(\mathbf{d})$ and two starting volumes

postulated to scale with the density times the power $3 / 2$ of the granular temperature. The agreement between the computed and the measured depth-averaged density values is good using one set of three parameters for the eight flows investigated (two starting volumes and four different chute inclinations). With regard to the model assumptions we interpret the evolution of density in a finite sized, dry granular avalanche as follows:

- The depth-averaged density is lower in the flow head and tail and higher in the flow body. In the flow head and tail, the flow height is smaller and thus the conduction of fluctuation energy from the bed surface to the free surface is important. In addition the normal stress is lower in the flow head and tail, allowing the granular material to dilate more for a given granular temperature.

- The depth-averaged density is lower for flows with large chute inclination. Two effects are competing. On the one hand the flow height is larger for flows with large chute inclination and less fluctuation energy is conducted to the free surface. On the other hand the shear rate and the slip velocity are larger for flows with large chute inclination resulting in larger production at the bed surface and in the flow bulk. All in all the last effect dominates.

- The depth-averaged density is lower for flows with small starting volume. The explanation follows from the previous points of discussion. The shear rate and slip velocity are similar or slightly larger whereas the flow height is smaller allowing for larger conduction of fluctuation energy over the flow depth and enhanced dilation (lower overburden pressure).

We now compare the terms in Eq. (19). For the flow with 301 starting volume and $29.5^{\circ}$ chute inclination, we find that the first conduction term is in average three times larger than the source term, the second conduction term is five times smaller and the decay term is five times larger. The smallest term in Eq. (19) (second conduction term) is thus 10 times larger than the three terms (time derivative, spatial gradient and normal stress terms) on the first line of Eq. (13). Indeed the source term is 50 times larger than the three terms on the first line according to the shallow flow scaling. Therefore we argue that the important terms for the computation of the granular temperature were taken into account. 
We note that Eq. (19) is valid only if the shear rate scales with $\frac{\sqrt{g L}}{H}$ as we assumed it when simplifying Eq. (11). In the case of a plug flow the shear rate would be close to zero and terms in Eq. (11) could not be neglected. The same remark holds for the conduction of $T$. In the case of zero spatial gradients of $T$ and $\rho$ in the $y$-direction additional terms should be accounted in Eq. (19).

The choice of boundary conditions for $T$ makes up a weakness of the model since they are not supported by direct measurements of the granular temperature. At the bed surface we put forward the evolution of the slip velocity with respect to the basal normal stress to set up the value of $T$. In [26] the authors derive the boundary condition $T(y=0) \propto u_{s}^{2}$ at a bumpy bed surface assuming low density and $u_{s} \ll \sqrt{T}$. At the free surface it is simplicity that dictates our choice of a zero spatial gradient of $T$ in the $y$-direction. An alternative boundary condition would be a zero conduction term at the free surface $\rho \frac{\partial^{2} T}{\partial y^{2}}+\frac{\partial \rho}{\partial y} \frac{\partial T}{\partial y}=0$. However using the condition of a zero spatial gradient, we obtain values of the conduction term close to zero for all flows at the free surface.

The simplified fluctuation energy equation in Eq. (19) also applies to steady and uniform flows. Indeed it does not include any time derivative or spatial gradients in the $x$-direction terms. Likewise the constitutive relations for the internal stresses and the decay coefficient are also valid in the case of steady and uniform flows. In contrast the mass and momentum equation in the $x$-direction for steady and uniform flows do not apply to unsteady flows. That is the reason why we do not solve the full system of governing equations since it would require that we compute the flow over time and space ( $x$ - and $y$-directions) and not only in the $y$-direction at the location of the camera. In $[27,28]$ the full system of governing equations is solved numerically for steady and uniform flows that is equivalent to computing the flow only in the $y$-direction. Density and granular temperature profiles are qualitatively similar to the ones in Fig. 9.

\section{Conclusions}

Dry granular avalanches in the frictional-collisional regime are generally modeled as continuum materials obeying mass and momentum conservation equations for two-dimensional, shallow flows. Their description is completed by constitutive relations specifying shear stress and density and by boundary conditions. Common constitutive relations assume a normal stress (Coulomb friction) and/or shear rate (power law rheology) dependent shear stress and constant density $[2,9,11,12]$. In the present work evidence of density variations in dense, sheared, dry granular avalanches is shown. Moreover the dependence of density on chute inclination and starting volume is quantified. The consequences of non-constant den-
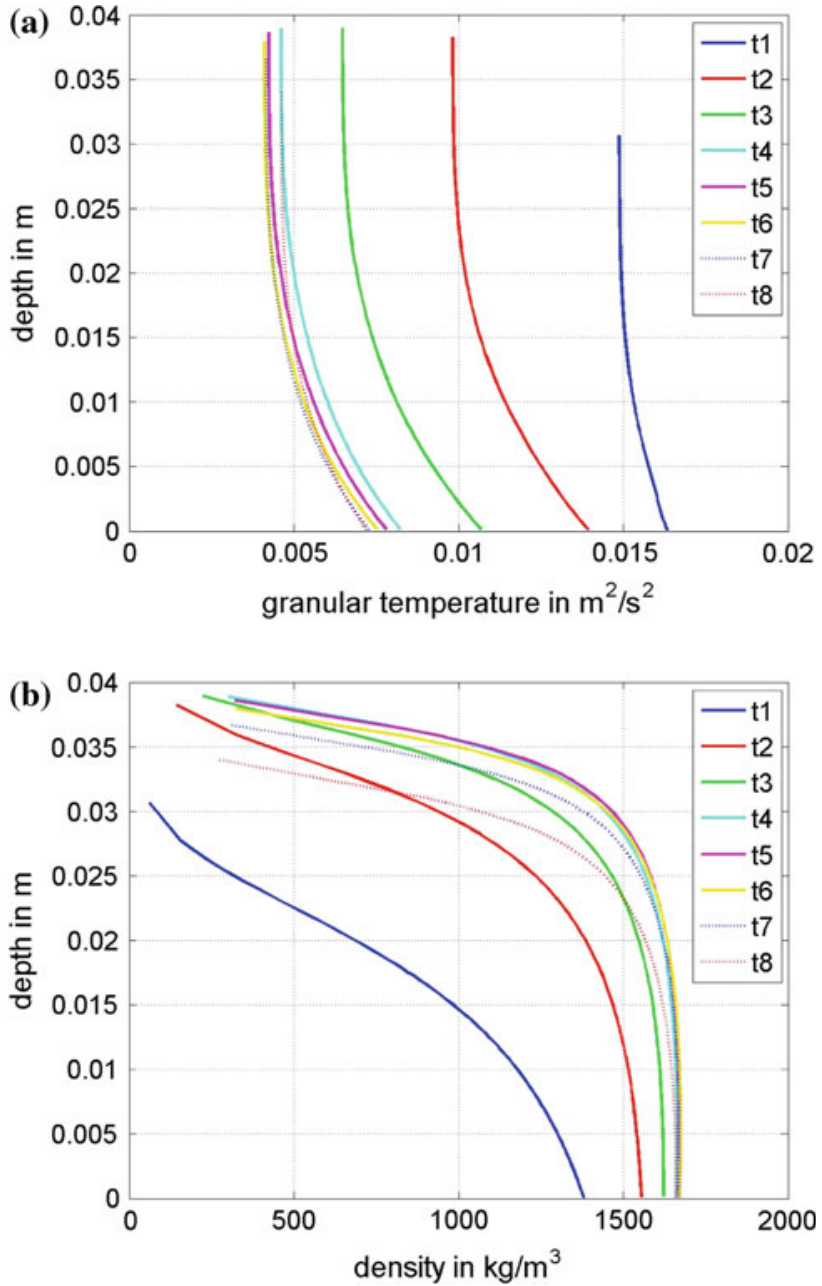

Fig. 9 Computed profiles of granular temperature (a) and density (b) versus flow depth at eight instants during the passing of the flow with $27^{\circ}$ chute inclination and 301 starting volume

sity for the modeling are wide-ranging. They include: extra terms in the mass and momentum conservation equations which make depth-averaging more difficult, another equation if fluctuation energy is taken into account and additional constitutive relations since the number of equations minus the number of unknown variables is increased. We add that non-constant density de facto brings together models assuming either normal stress or shear rate dependent shear stress since the density factor in Eq. (15) includes implicitly the normal stress dependence.

The present model has interesting implications for flows with different boundary conditions or geometry. In this work, the computed granular temperature is maximum at the bed surface. However for flows with low slip velocity compared to the shear rate (very rough bed surface, large normal stress, lubricated grains) the granular temperature would be larger in the flow bulk than at the bed surface, modifying the shape of the velocity and density profiles. A limitation of the model 
concerns frictional flows characterized by low shear rate, large normal stress and high density. Enduring and multiparticle contacts dominate in the frictional regime and the constitutive relations proposed here would likely not be valid. In particular the contact rate would not scale with the shear rate and the energy lost per contact would not be proportional to the shear rate squared. Future work shall aim at the combined measurement of velocity, density and granular temperature profiles. Such experimental data would enable the direct derivation of constitutive relations and the verification of their validity over the flow variable space.

\section{Appendix}

The interval $[0, h]$ is discretized in $N$ elements. The vector $y$ with size $N+1$ contains the $y$-coordinates of the elements edges. In a first step Eq. (18) is integrated for $n=N$ to 1 yielding the normal stress vector with size $N+1$ :

$p^{n}=p^{n+1}+\rho^{n} g \cos \theta\left(y^{n+1}-y^{n}\right)$.

with the boundary conditions $p^{N+1}=0$. In a second step Eq. (19) is integrated over the flow depth. First the vector $\frac{\partial T}{\partial y}$ with size $N+1$ is computed for $n=N$ to 1 , second the vector $T$ with size $N+1$ is calculated for $n=2$ to $N+1$ :

$$
\begin{aligned}
&{\frac{\partial T^{n}}{\partial y}=}^{n} \frac{\partial T^{n+1}}{\partial y}-\left(y^{n+1}-y^{n}\right)\left(-\frac{\tau_{0}}{\kappa l^{n+1}}\left(d \frac{\partial u}{\partial y}\right)^{3}\right. \\
&\left.-\frac{1}{\rho^{n+1}} \frac{\partial \rho^{n+1}}{\partial y} \frac{\partial T^{n+1}}{\partial y}+\frac{\gamma_{0}}{\kappa l^{n+1}} T^{n+1 \frac{3}{2}}\right) \\
& T^{n+1}= T^{n}+\left(y^{n+1}-y^{n}\right) \frac{\partial T^{n}}{\partial y}
\end{aligned}
$$

with the boundary conditions $\frac{\partial T}{\partial y}^{N+1}=0$ and $T^{1}=\xi \sqrt{u_{s}}$. In a third step, the normal stress and the granular temperature are evaluated at the centre of the elements (vectors with size $N$ ) and a new density vector with size $N$ is calculated. The size of the elements 1 to $N$ i.e. the vector $y$ is modified with respect to the new density vector so that the mass of each element is conserved.

\section{References}

1. Christen, M., Kowalski, J., Bartelt, P.: Numerical simulation of avalanches in three-dimensional terrain. Cold Reg. Sci. Technol. 63, 1-14 (2010)

2. Savage, S.B., Hutter, K.: The motion of a finite mass of granular material down a rough incline. J. Fluid Mech. 199, 177-215 (1989)

3. Louge, M., Steiner, R., Keast, S., Decker, R., Dent, J., Schneebeli, M.: Application of capacitance instrumentation to the measurement of density and velocity of flowing snow. Cold Reg. Sci. Technol. 25(1), 47-63 (1997)
4. Dent, J.D., Burrell, K.J., Schmidt, D.S., Louge, M.Y., Adams, E.E., Jazbutis, T.G.: Density, velocity and friction measurements in a dry-snow avalanche. Ann. Glaciol. 26, 247-252 (1998)

5. Sovilla, B., Schaer, M., Kern, M., Bartelt, P.: Impact pressures and flow regimes in dense snow avalanches observed at the Vallée de la Sionne test site. J. Geophys. Res. 113, F01010 (2008)

6. Bartelt, P., McArdell, B.: Granulometric investigations of snow avalanches. J. Glaciol. 55(193), 829-833 (2009)

7. Bagnold, R.: Experiments on a gravity-free dispersion of large solid spheres in a newtonian fluid under shear. Proc. R. Soc. Lond. Ser. A Math. Phys. Sci. 225, 49-63 (1954)

8. Boyer, F., Guazzelli, E., Pouliquen, O.: Unifying suspension and granular rheology. Phys. Rev. Lett. 107, 188301 (2011)

9. Pouliquen, O.: Scaling laws in granular flows down rough inclined planes. Phys. Fluids 11(3), 542-548 (1999)

10. Savage, S.B.: Gravity flow of cohesionless granular materials in chutes and channels. J. Fluid Mech. 92(1), 53-96 (1979)

11. Ancey, C., Evesque, P.: Frictional-collisional regime for granular suspension flows down an inclined channel. Phys. Rev. E 62, 8349 (2000)

12. Andreotti, B., Daerr, A., Douady, S.: Scaling laws in granular flows down a rough plane. Phys. Fluids 14(1), 415-418 (2002)

13. Ancey, C.: Dry granular flows down an inclined channel: experimental investigations on the frictional-collisional regime. Phys. Rev. E 65, 011304 (2001)

14. Silbert, L.E., Ertas, D., Grest, G.S., Halsey, T.C., Levine, D., Plimpton, S.J.: Granular flow down an inclined plane: Bagnold scaling and rheology. Phys. Rev. E 64, 051302 (1997)

15. Haff, P.K.: Grain flow as a fluid-mechanical phenomenon. J. Fluid. Mech. 134, 401-430 (1983)

16. Jenkins, M.T., McTigue, D.F.: Transport processes in concentrated suspensions: the role of particle fluctuations. Inst. Math. Appl. 26, 70 (1990)

17. Jenkins, J.T., Richman, M.W.: Kinetic theory for plane flows of a dense gas of identical, rough, inelastic, circular disks. Phys. Fluids 28, 3485-3489 (1985)

18. Goldhirsch, I., Tan, M.L.: The single particle distribution function for rapid granular shear flows of smooth inelastic disks. Phys. Fluids 8, 1752-1763 (1996)

19. Savage, S.B.: Granular flows down rough inclines-review and extension. In: Jenkins, J. T., Satake, M. (eds.) Mechanics of granular materials: new models and constitutive relations, pp. 261-282. Elsevier, Amsterdam (1983)

20. Schaefer, M., Bugnion, L., Kern, M., Bartelt, P.: Position dependent velocity profiles in granular avalanches. Granul. Matter 12(3), 327$336(2010)$

21. Jaeger, H.M., Nagel, S.R.: Granular solids, liquids, and gases. Rev. Mod. Phys. 68(4), 1259-1274 (1990)

22. D’Anna, G., Mayor, P., Barrat, A., Loreto, V., Nori, F.: Observing brownian motion in vibration-fluidized granular matter. Nature 424, 909-912 (2003)

23. Goldhirsch, I.: Rapid granular flows. Annu. Rev. Fluid. Mech. 35, 267-297 (2003)

24. Son, R., Perez, J.A., Voth, G.A.: Experimental measurements of the collapse of a two-dimensional granular gas under gravity. Phys. Rev. E, 041302 (2008)

25. Landau, L., Lifchitz, E.: Mécanique des fluides, Editions de Moscou, Collection Physique théorique, vol. 6 (1971)

26. Richman, M.W.: Boundary conditions based upon a modified Maxwellian velocity distribution for flows of identical, smooth, nearly elastic spheres. Acta Mech. 75, 227-240 (1988)

27. Forterre, Y., Pouliquen, O.: Stability analysis of rapid granular chute flows: formation of longitudinal vortices. J. Fluid Mech. 467, 361-387 (2002)

28. Woodhouse, M.J., Hogg, A.J., Sellar, A.A.: Rapid granular flows down inclined planar chutes. J. Fluid Mech. 652, 427-460 (2010) 